\title{
Exotic Quarkonium states at CMS
}

\author{
Leonardo Cristella* \\ INFN Section of Bari \\ E-mail: leonardo.cristella@ba.infn.it
}

The studies of the production of the $X(3872)$, either prompt or from B hadron decays, and of the $J / \psi \phi$ mass spectrum in B hadron decays have been carried out by using $p p$ collisions at $\sqrt{s}=7 \mathrm{TeV}$ collected with the CMS detector at the LHC. The cross-section ratio of the $X(3872)$ with respect to the $\psi(2 S)$ in the $J / \psi \pi^{+} \pi^{-}$decay channel and the fraction of $X(3872)$ coming from B-hadron decays are measured as a function of transverse momentum $\left(p_{\mathrm{T}}\right)$, covering unprecedentedly high values of $p_{\mathrm{T}}$. For the first time, the prompt production cross section for the $X(3872)$ times the unknown branching fraction for the decay of $X(3872) \rightarrow J / \psi \pi^{+} \pi^{-}$is extracted differentially in $p_{\mathrm{T}}$ and compared to theoretical predictions based on the Non-Relativistic QCD (NRQCD) factorization approach. The dipion invariant-mass spectrum of the $J / \psi \pi^{+} \pi^{-}$ system in the $X(3872)$ decay is also investigated.

The search for resonance-like structures in the $B_{s}^{0} \pi^{ \pm}$invariant mass spectrum do not show any unexpected result. An upper limit on the relative production of the claimed $X(5568)$ and $B_{S}$ multiplied by the unknown branching fraction of the decay $X(5568) \rightarrow B_{s} \pi^{ \pm}$is estimated to be $3.9 \%$ at $95 \% \mathrm{CL}$ in the most conservative case.

The 39th International Conference on High Energy Physics (ICHEP2018)

4-11 July, 2018

Seoul, Korea

${ }^{*}$ Speaker. 


\section{Measurements of the $X(3872)$ state}

The analysis is performed on the data recorded by the CMS experiment in 2011, corresponding to an integrated luminosity of $4.8 \mathrm{fb}^{-1}$. The $X(3872)$ is observed using the decays into $J / \psi \pi^{+} \pi^{-}$, with the subsequent decay of the $J / \psi$ into a pair of muons. The analysis is thus performed in the kinematic range of $p_{\mathrm{T}}$ of the $J / \psi \pi^{+} \pi^{-}$system between 10 and $50 \mathrm{GeV}$ and the rapidity within $|y|<1.2$, collecting about $12000 X(3872)$ candidates. The event selection and the event simulations, used to determine acceptances and efficiencies, are described in detail in Ref.[4]. The $X(3872)$ is assumed to be an unpolarized state and its $J^{P C}$ is fixed to $1^{++}$as favoured by the existing studies [5].

\subsection{The prompt $X(3872)$ production cross section}

The cross section times branching fraction for prompt $X$ (3872) production is determined from the measurement of the cross section ratio $R$ and the nonprompt fraction, combined with a previous result of the prompt $\psi(2 S)$ cross section obtained in CMS [6]. By means of this combination, the differential cross section for prompt $X(3872)$ production times the branching fraction is obtained as a function of $p_{\mathrm{T}}$, in the rapidity region $|y|<1.2$, as shown in Fig.1. The $X(3872)$ and $\psi(2 S)$ states are assumed to be unpolarized and no cancellation of systematic uncertainties is assumed in the combination. The main sources of systematic uncertainty are related to the measurement of $R$ and of the prompt $\psi(2 S)$ cross section [6]. The differential cross section for prompt $X(3872)$ production in $p p$ collisions at $\sqrt{s}=7 \mathrm{TeV}$ has also been predicted with a calculation made within the NRQCD factorization formalism [7]. The comparison of this prediction with the data is shown in Fig. 1 and demonstrates that, while the shape is reasonably described, the predicted cross section is much larger than the measured one. The integrated prompt $X(3872)$ cross section times branching fraction for the kinematic region $10<p_{\mathrm{T}}<30 \mathrm{GeV}$ and $|y|<1.2$ is also determined to be

$$
\begin{gathered}
\sigma^{\text {prompt }}(p p \rightarrow X(3872)+\text { anything }) \cdot B\left(X(3872) \rightarrow J / \psi \pi^{+} \pi^{-}\right)= \\
1.06 \pm 0.11 \text { (stat. }) \pm 0.15 \text { (syst.) nb. }
\end{gathered}
$$

This measured value is below the theoretical prediction for the prompt $X(3872)$ cross section times branching fraction in the same kinematic region, which is $4.01 \pm 0.88 \mathrm{nb}$ [7].

\subsection{Search for the $X_{b}$ state}

Heavy-quark symmetry suggests the existence of a hidden-beauty partner of the $X(3872)$ referred to as $X_{b}$, which should be produced in $p p$ collisions. CMS looked for the $X_{b}$ state [9] in events with a $\Upsilon(1 \mathrm{~S})$ candidate and two additional opposite-charged tracks, assumed to be pions. A common vertex has been fitted with muons and pions, and the invariant mass has been computed by constraining the dimuon mass to the $\Upsilon(1 \mathrm{~S})$.

The ratio R of cross-sections for $X_{b}$ and $\Upsilon(2 S)$ was estimated for different hypotheses about $X_{b}$ mass. The search has been performed in two regions, [10.05, 10.31] GeV and [10.40, 10.99] GeV, to exclude the $\Upsilon(2 S)$ and $\Upsilon(3 S)$; the mass spectrum has been fitted with a gaussian function for the resonances and a polynomial for the background. The $X_{b}$ mass has been shifted by $10 \mathrm{MeV}$ steps, while its width has been assumed to be small, and the resolution was taken from simulation. The 


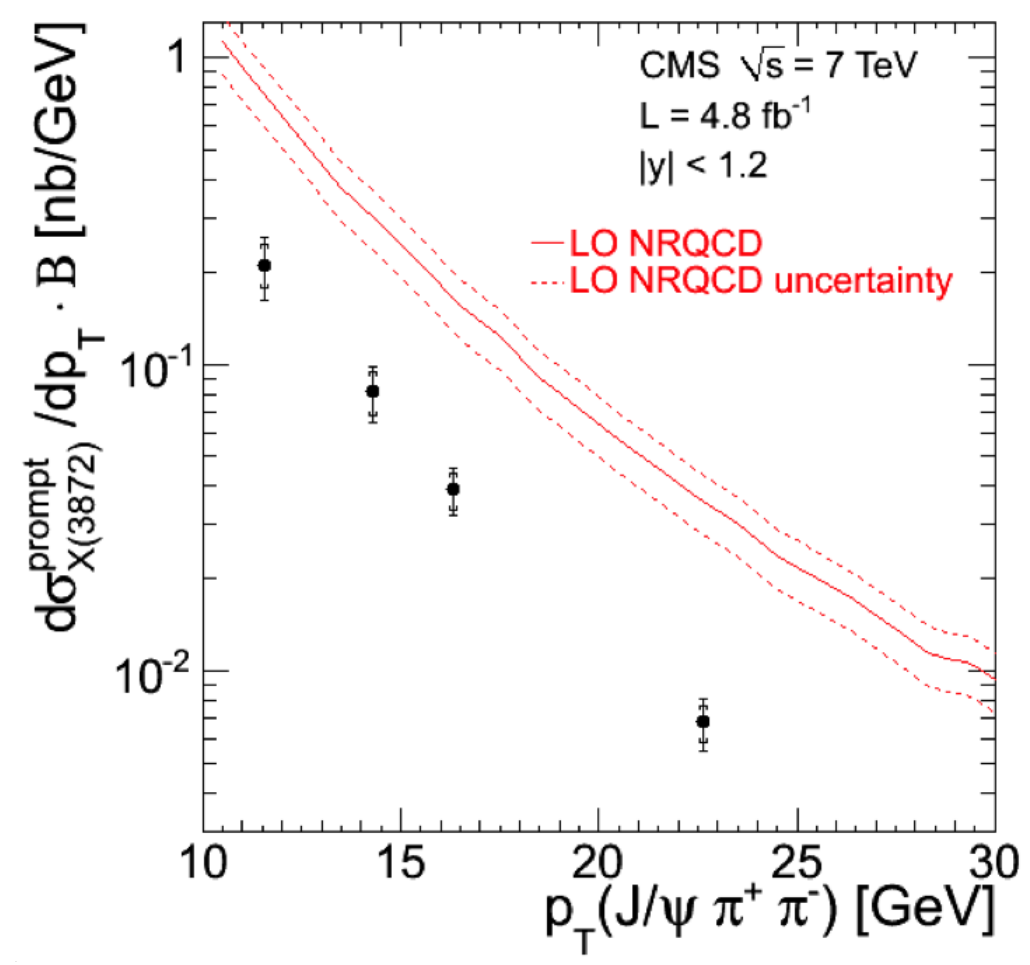

Figure 1: Measured differential cross section for prompt $X(3872)$ production times branching fraction of $X(3872) \rightarrow J / \psi \pi^{+} \pi^{-}$ as a function of $p_{\mathrm{T}}$. The inner error bars indicate the statistical uncertainty while the outer error bars represent the total uncertainty. Theoretical prediction as explained in the text is shown by the solid line with the dotted lines representing the related uncertainty.

ratio $\mathrm{R}$ was then given by the ratio of the observed candidates scaled with the ratio of efficiencies. Signal strength, P-values and cross section limits have been computed versus $X_{b}$ mass. Results are shown in Figure 2.
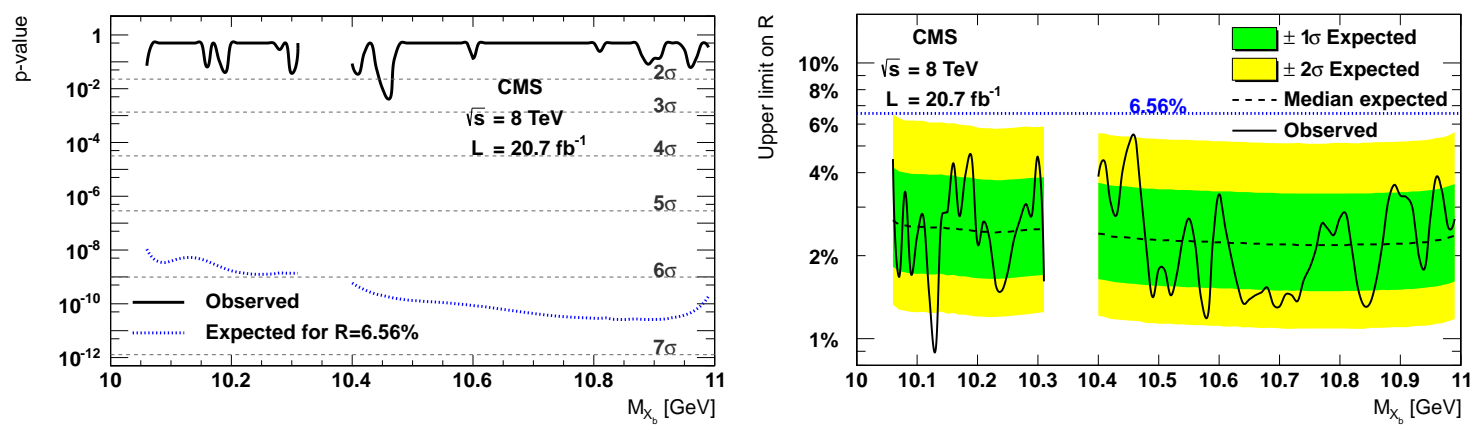

Figure 2: P-value (left) and upper limit at $95 \%$ confidence level on R (roght) as a function of the assumed $X_{b}$ mass. The lines at $6.56 \%$ correspond to the expectations for the analogous $X(3872)$ decay to $J / \psi \pi^{+} \pi^{-}$.

\section{Search for the $X(5568)$ in the CMS data}

Recently the $\mathrm{D} \varnothing$ Collaboration claimed the evidence of a narrow state in the $B_{s}^{0} \pi^{ \pm}$invariant mass spectrum with mass $m=\left(5567 \pm 2.9_{-1.9}^{+0.9}\right) \mathrm{MeV}$ and $\Gamma=\left(21.9 \pm 6.4_{-2.5}^{+5.0}\right) \mathrm{MeV}$ [10], thus raising a lot of interest within the community that studies exotic hadrons [11] and triggered this search at several hadron collider experiments, including CMS [12] and LHCb [13]. The CMS search for resonance-like structures in the $B_{s}^{0} \pi^{ \pm}$invariant mass spectrum was performed using an integrated 
luminosity of $19.7 \mathrm{fb}^{-1}$ of pp collisions at $\sqrt{s}=8 \mathrm{TeV}$. The $B_{s}^{0}$ candidates are recostructed in the decay chain $B_{s}^{0} \rightarrow J / \psi \phi, J / \psi \rightarrow \mu^{+} \mu^{-}, \phi \rightarrow K^{+} K^{-}$. The $B_{s}^{0} \pi^{ \pm}$invariant mass distributions do not show any unexpected structures for different kinematic requirements imposed to the $\pi^{ \pm}, B_{s}^{0}$ and $B_{s}^{0} \pi^{ \pm}$candidates. An upper limit on the relative production of $X(5568)$ and $B_{s}$ multiplied by the branching fraction of the decay $X(5568) \rightarrow B_{s} \pi^{ \pm}$is estimated to be $1.1 \%$ at $95 \%$ CL in the most conservative case. Results are shown in Figure 3.
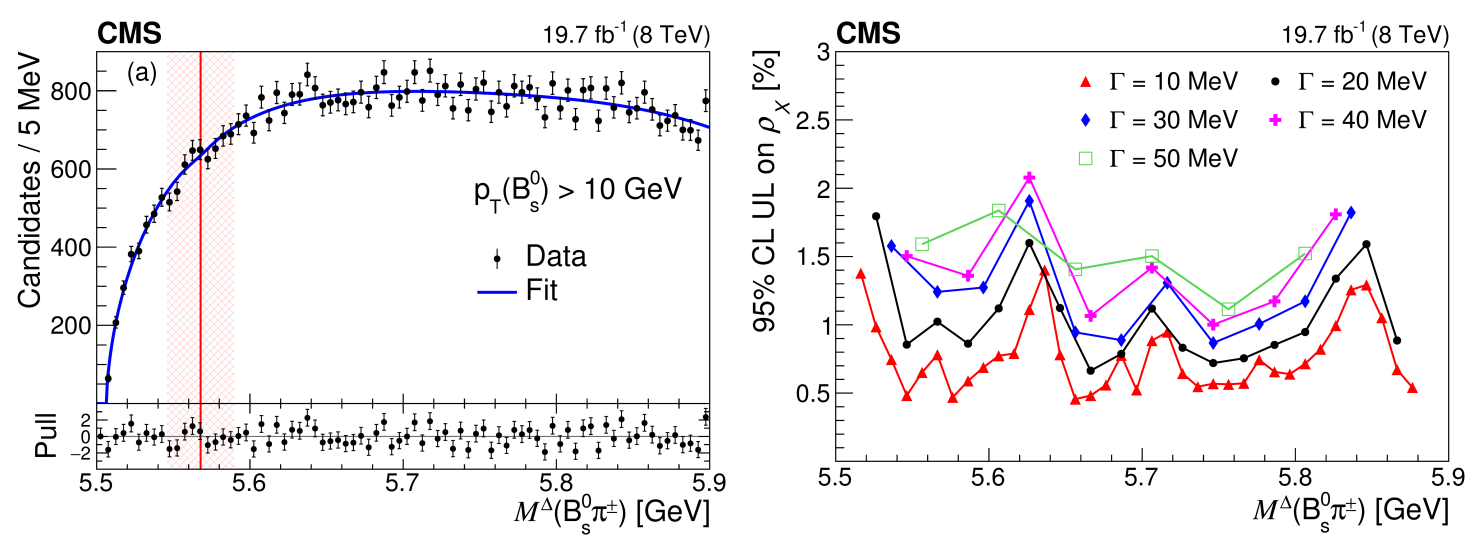

Figure 3: (left) Mass distribution of events in the $B_{s}$ signal region (black points with error bars) with fit results superimposed (blue line). (right) Distribution of the upper limit on the relative production of $X(5568)$ and $B_{s}$, for different values of the $X(5568)$ width.

\section{References}

[1] Belle Collaboration, Phys. Rev. Lett. 91, 262001 (2003)

[2] N. Brambilla et al., Eur. Phys. J. C 71, 1534 (2011)

S. L. Olsen, Front. Phys. 10, 2, 121-154 (2015)

[3] CMS Collaboration, JINST 3, S08004 (2008)

[4] CMS Collaboration, JHEP 04,154 (2013)

[5] LHCb Collaboration, Phys. Rev. Lett. 110, 222001 (2013)

LHCb Collaboration, Phys. Rev. D 92, 011102 (2015)

[6] CMS Collaboration, JHEP 02, 011 (2012)

[7] P. Artoisenet and E. Braaten, Phys. Rev. D 81, 114018 (2010)

[8] CDF Collaboration, Phys. Rev. Lett. 96, 102002 (2006)

[9] CMS Collaboration, Phys. Lett. B 727, 57 (2013)

[10] V. M. Abazov et al., Phys. Rev. Lett., 117(2), 022003 (2016)

[11] T.J. Burns and E.S.Swanson, Phys. Lett. B 760, 627 (2016) F.-K. Guo et al., Commun. Theor. Phys. 65(5), 593 (2016)

A.Ali et al., Phys. Rev. D 94, 034036 (2016)

[12] CMS Collaboration, Phys. Rev. Lett. 117202005 (2018) (2016)

[13] LHCb Collaboration, Phys. Rev. Lett. 117, 152003 (2016) 